\title{
Relation between luminous efficiency function and color matching functions
}

\author{
Hirohisa YAGUCHI*
}

\begin{abstract}
Color matching functions and luminous efficiency functions by flicker photometry for a $2^{\circ}$ foveal field, were measured for two observers at a retinal illuminance of 100 trolands. The synthetic luminous efficiency function determined as a linear combination of color matching functions was compared with the measured one for a given observer. Taking into account the uncertainties of measurements, the synthetic functions were in good agreement with the measured one.
\end{abstract}

\section{Introduction}

Analyzing the relationships among various visual functions; color matching functions, luminous efficiency function, chromatic valence functions, wavelength discrimination, foveal threshold, and so on, gives us a great deal of knowledge of how the visual system processes color information. However, inter-observer deviation of visual functions caused by individual differences of macular pigment and lens absorption is an obstacle in analyzing the data. To make a quantitative model of color vision or to test an existing color vision model, therefore, it is appropriate to measure a set of visual functions for a given observer and apply it to the models. Among various visual functions, color matching functions and luminous efficiency function are the most important ones, because they provide not only the basis of color vision models but also the basis of colorimetry and photometry.

The 1931 CIE Standard Observer color matching functions were derived from Wright's and Guild's colorimetric data (not color matching functions but chromaticity coordinates of spectral colors) and the $1924 \mathrm{CIE} V(\lambda)$ function. This derivation relies on the assumption that the $V(\lambda)$ function is a linear combination of color matching functions. There have been several studies that examine the validity of this important assumption.

The first study was made by Stiles. ${ }^{1)} \mathrm{He}$

* Department of Image Science and Engineering Faculty of Engineering, Chiba University

1-33 Yayoi-cho, Chiba, 260 Japan measured $2^{\circ}$ color matching functions for 10 observers. He also determined the luminous efficiency function for a $2^{\circ}$ field in the blue end of spectrum by heterochromatic brightness matching for 28 observers including the 10 observers for whom color matching functions had also been measured. A linear combination of the mean color matching functions using the observer's own direct measured luminous efficiencies at the primary colors, were compared with the mean of his direct measured luminous efficiency function. The synthetic luminous efficiency function was generally in good agreement with the directly measured one, but the values were relatively low in the blue region. Stiles suggested that a similar test should be applied to the complete luminous efficiency function determined for the same observers by some acceptable procedure of heterochromatic photometry, such as flicker photometry for example.

Later on, Sperling ${ }^{2)}$ carried out a similar test with six observers. He measured the luminous efficiency functions using two methods, heterochromatic brightness matching and flicker photometry. He compared each of the luminous efficiency functions with a linear combination of their average color matching functions weighted by the respective luminous efficiencies at the primaries. Sperling found that deviations of the synthetic luminous efficiency function from the real one were large through part of the spectrum for brightness matching but small for flicker photometry. His results imply that brightness matching does not obey the additivity law, whereas the flicker photometry does. Al- 
though Sperling concluded that the deviations found using the flicker photometry did not allow rejection of the linearity hypothesis between color matching functions and the luminous efficiency function, Estevez ${ }^{3)}$ pointed out that the differences appeared to be systematic and they were similar to Stiles's results.

Richards and Luria $^{4)}$ also measured color matching functions and luminous efficiency functions by flicker photometry for three observers at three luminance levels in the mesopic region. They found no significant difference between the synthetic luminous efficiency function and the measured one at all luminance levels.

In the present study, we re-examined this relationship using the same observers.

\section{Methods and results}

\subsection{Color matching functions}

The color matching experiments were done on the NRC Trichromator originally designed by Stiles. A schematic view and details of the Trichromator are described elsewhere. ${ }^{5), 6)}$ Color matching functions were obtained by the maximum saturation method. A $2^{\circ}$ bipartite field was displayed in a dark surround with Maxwellian view. The upper half of the bipartite field provided two of the primary stimuli of the Trichromator at wavelength $\lambda_{R}=645.2 \mathrm{~nm}$ $\left(15,500 \mathrm{~cm}^{-1}\right.$ in wavenumber $), \lambda_{G}=526.3 \mathrm{~nm}$ $\left(19,000 \mathrm{~cm}^{-1}\right)$, and $\lambda_{B}=444.4 \mathrm{~nm}(22,500$ $\left.\mathrm{cm}^{-1}\right)$. The lower half provided the monochromatic test stimuli in the range $408 \mathrm{~nm}$ to 690 $\mathrm{nm}$ in $250-\mathrm{cm}^{-1}$ wavenumber steps, and a desaturating primary. The observer controlled the radiance of three primary stimuli to make a color match between the upper half and the lower half of the field by the method of adjustment. The retinal illuminances of the test stimuli between $417 \mathrm{~nm}$ and $690 \mathrm{~nm}$ were 100 $\mathrm{Td}$. In the shorter wavelength region however, they were $75 \mathrm{Td}$ for $412 \mathrm{~nm}$ and $35 \mathrm{Td}$ for 408 $\mathrm{nm}$ because of insufficient light.

The test spectrum was divided into two sets, one was from $408 \mathrm{~nm}$ to $690 \mathrm{~nm}$ in $500-\mathrm{cm}^{-1}$ wavenumber steps, and the other from $412 \mathrm{~nm}$ to $678 \mathrm{~nm}$ in $500-\mathrm{cm}^{-1}$ steps. Each set was run through in one of two directions, that is, from blue to red or from red to blue, in a separate experimental session. One match was made at each test wavelength. It took about one hour to complete one session. Two observers; HY (34 years) and ZF (40 years), joined this experiments. Four experimental sessions were repeated for each observer.

Color matching functions were determined directly from the radiant powers of a test stimulus and three primaries. If radiant powers $R(\lambda)$, $G(\lambda)$, and $B(\lambda)$ of the three primaries are required to match a test monochromatic stimulus (wavelength, $\lambda$ ) of radiant power $L(\lambda)$, the color matching functions $\bar{r}(\lambda), \bar{g}(\lambda)$, and $\bar{b}(\lambda)$ are determined as follows,

$$
\begin{aligned}
& \bar{r}(\lambda)=R(\lambda) / L(\lambda) \\
& \bar{g}(\lambda)=G(\lambda) / L(\lambda) \\
& \bar{b}(\lambda)=B(\lambda) / L(\lambda) .
\end{aligned}
$$

The amount of the desaturating primary stimulus is given a negative sign.

The raw data of the color matching functions from the four sessions were plotted on a logarithmic scale, and then smoothed and normalized so that the color matching function was unity at the wavelength of the respective primary. The resultant color matching functions for two observers are shown in Table 1 and Fig. 1.

\subsection{Luminous efficiency function by flicker photometry}

The flicker photometry experiment was also done on the same Trichromator. In this experiment, a $2^{\circ}$ full field was presented in a dark surround. A reference white stimulus was provided by mixing three primary stimuli of the Trichromator to give the chromaticity coordinates of $\mathrm{D}_{65}$ white $(x=0.313, y=0.329)$ and a retinal illuminance of $100 \mathrm{Td}$. The test stimulus was one of the monochromatic stimuli, from $408 \mathrm{~nm}$ to $690 \mathrm{~nm}$ in $250-\mathrm{cm}^{-1}$ wavenumber steps. The reference stimulus and the test stimulus were presented alternately. The flicker frequency was set at $20 \mathrm{~Hz}$ throughout the test wavelength region. The observer adjusted the radiance of the test stimulus to determine the minimum flicker point. In some wavelength regions, for example in the green-yellow region, flicker disappeared completely over a certain small range of test radiances. In this case, the observer determined the middle point of the flicker disappearance range. Three repeats were made at each test wavelength in each session. 

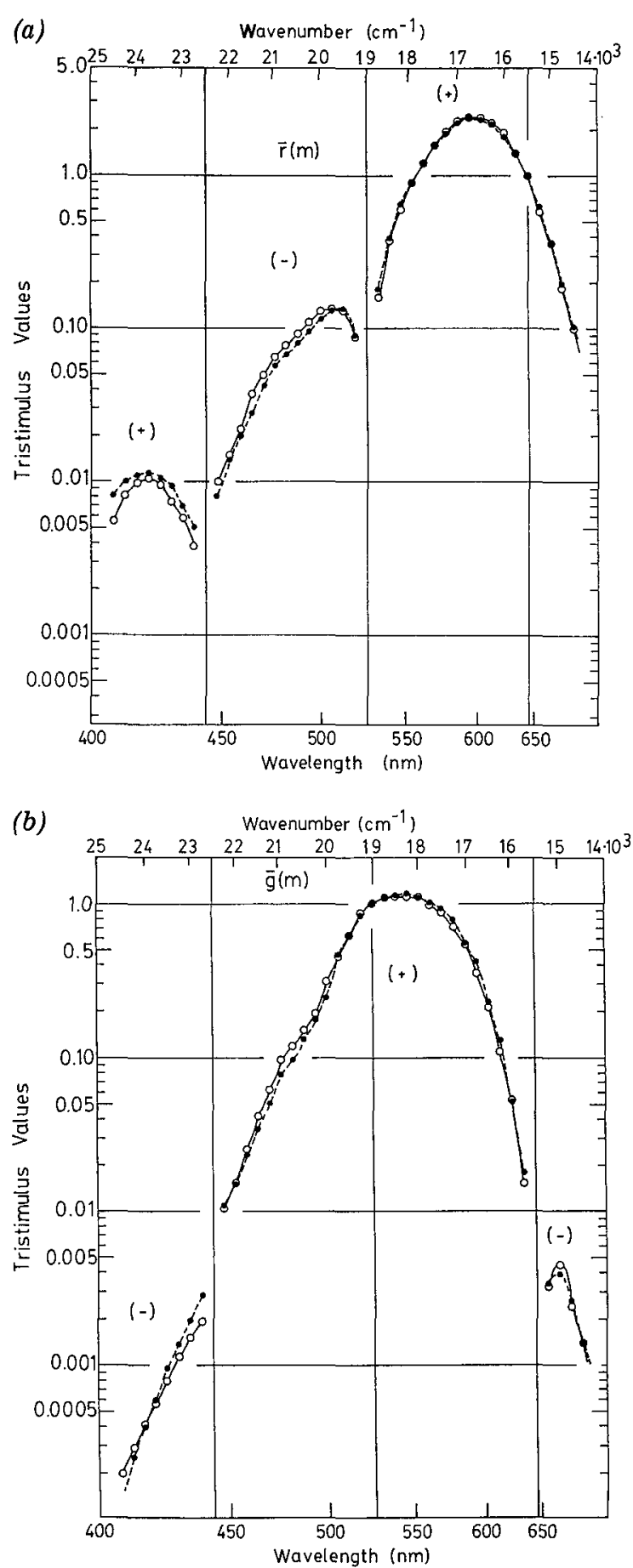

There were four sessions for one observer (HY), and two sessions for another (ZF). The luminous efficiency function was determined by the reciprocal of the energy of the test stimulus required to provide minimum flicker.

The luminous efficiency functions by flicker photometry for the same two observers are shown in Table 1. The values were normalized to unity at $555.6 \mathrm{~nm}$. The luminous efficiency functions are plotted in Fig. 2. Vertical bars in HY's plots show \pm 1 standard deviation. Standard deviations were not calculated for ZF's results because of insufficient data.

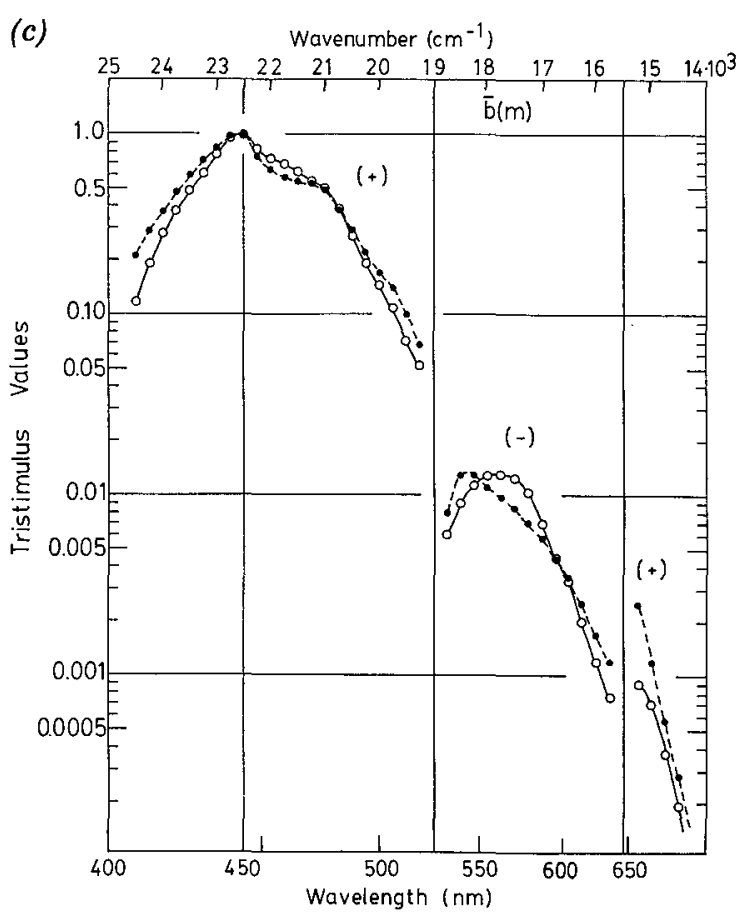

Fig. 1 Color matching functions, open circles: observer $H Y$, closed circles: observer $Z F$. (a): $\bar{r}(\lambda)$, (b): $\bar{g}(\lambda)$, and (c): $\bar{b}(\lambda)$.

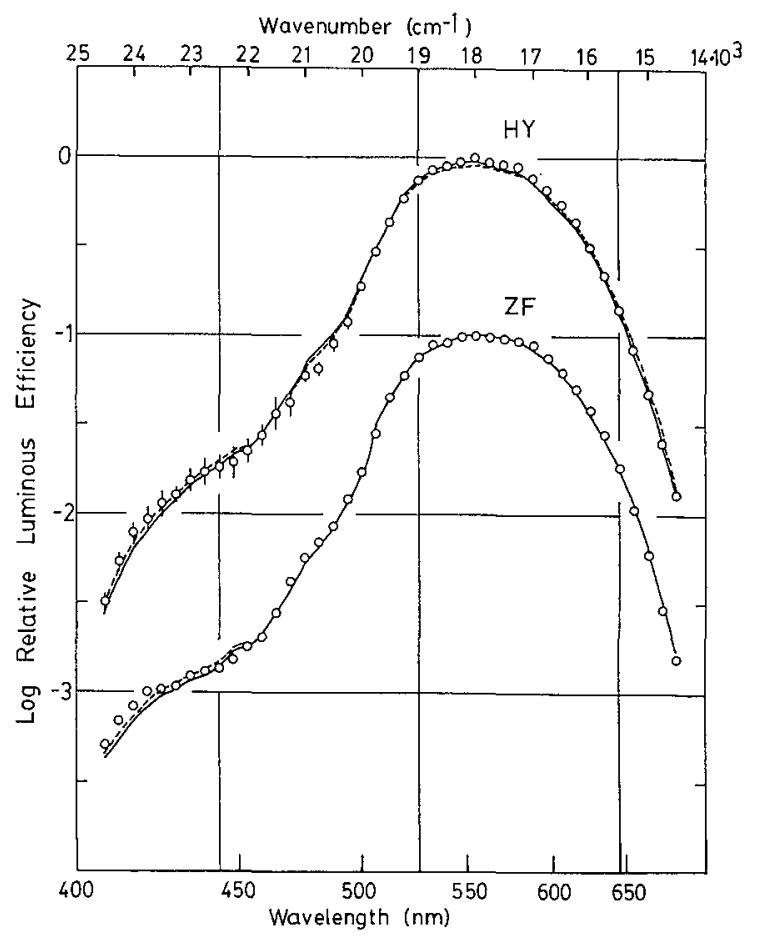

Fig. 2 Luminous efficiency functions for two observers. $Z F$ 's ones are displaced vertically by one logarithmic unit. Open circles show the experimental data obtained by flicker photometry, solid curve shows a linear combination of color matching functions weighted by the luminous efficiencies at the respective primaries, and the dashed curve shows that weighted by coefficients minimizing the deviations from the experimental data. 
Table 1. Color matching functions by maximum saturation method and luminous efficiency functions by flicker photometry

\begin{tabular}{|c|c|c|c|c|c|c|c|c|}
\hline \multirow{3}{*}{$\begin{array}{c}\text { Subject } \\
\text { Wave- } \\
\text { number } \\
\text { m } \\
(\mathrm{cm}-1)\end{array}$} & \multicolumn{4}{|c|}{ HY } & \multicolumn{4}{|c|}{$\mathrm{ZF}$} \\
\hline & \multicolumn{3}{|c|}{$\begin{array}{l}\text { Color matching } \\
\text { functions }\end{array}$} & \multirow{2}{*}{$\begin{array}{c}\text { Luminosity } \\
\text { functions } \\
V(\mathrm{~m})\end{array}$} & \multicolumn{3}{|c|}{$\begin{array}{l}\text { Color matching } \\
\text { functions }\end{array}$} & \multirow{2}{*}{$\begin{array}{c}\text { Luminosity } \\
\text { functions } \\
V(\mathrm{~m})\end{array}$} \\
\hline & $\bar{r}(\mathrm{~m})$ & $\bar{g}(\mathrm{~m})$ & $\bar{b}(\mathrm{~m})$ & & $\bar{r}(\mathrm{~m})$ & $\bar{g}(\mathrm{~m})$ & $\bar{b}(\mathrm{~m})$ & \\
\hline 14500 & 0.0982 & -0.00137 & 0.00019 & 0.0131 & 0.104 & -0.00140 & 0.00028 & 0.0158 \\
\hline 14750 & 0.180 & -0.00234 & 0.00037 & 0.0252 & 0.195 & -0.00260 & 0.00056 & 0.0300 \\
\hline 15000 & 0.360 & -0.00440 & 0.00070 & 0.0483 & 0.360 & -0.00390 & 0.00120 & 0.0601 \\
\hline 15250 & 0.584 & -0.00321 & 0.00090 & 0.0855 & 0.630 & -0.00340 & 0.00250 & 0.107 \\
\hline 15500 & 1.00 & 0.00000 & 0.00000 & 0.141 & 1.00 & 0.00000 & 0.00000 & 0.185 \\
\hline 15750 & 1.38 & 0.0151 & -0.00076 & 0.224 & 1.40 & 0.0180 & -0.00120 & 0.282 \\
\hline 16000 & 1.92 & 0.0532 & -0.00120 & 0.320 & 1.80 & 0.0520 & -0.00170 & 0.390 \\
\hline 16250 & 2.23 & 0.111 & -0.00199 & 0.443 & 2.20 & 0.130 & -0.00250 & 0.512 \\
\hline 16500 & 2.37 & 0.212 & -0.00331 & 0.553 & 2.36 & 0.230 & -0.00340 & 0.622 \\
\hline 16750 & 2.39 & 0.349 & -0.00448 & 0.671 & 2.40 & 0.420 & -0.00440 & 0.757 \\
\hline 17000 & 2.26 & 0.534 & -0.00697 & 0.780 & 2.20 & 0.560 & -0.00580 & 0.893 \\
\hline 17250 & 1.93 & 0.715 & -0.0105 & 0.889 & 1.90 & 0.790 & -0.00700 & 0.948 \\
\hline 17500 & 1.57 & 0.869 & -0.0125 & 0.929 & 1.58 & 0.930 & -0.00840 & 0.962 \\
\hline 17750 & 1.21 & 0.990 & -0.0129 & 0.942 & 1.20 & 1.03 & -0.00980 & 0.979 \\
\hline 18000 & 0.902 & 1.10 & -0.0127 & 1.00 & 0.900 & 1.11 & -0.0110 & 1.00 \\
\hline 18250 & 0.608 & 1.13 & -0.0113 & 0.955 & 0.660 & 1.17 & -0.0128 & 0.991 \\
\hline 18500 & 0.375 & 1.13 & -0.00896 & 0.887 & 0.390 & 1.14 & -0.0128 & 0.908 \\
\hline 18750 & 0.161 & 1.11 & -0.00598 & 0.845 & 0.180 & 1.09 & -0.00800 & 0.895 \\
\hline 19000 & 0.00000 & 1.00 & 0.00000 & 0.741 & 0.00000 & 1.00 & 0.00000 & 0.757 \\
\hline 19250 & -0.0771 & 0.864 & 0.0524 & 0.593 & -0.0880 & 0.830 & 0.0680 & 0.608 \\
\hline 19500 & -0.130 & 0.625 & 0.0712 & 0.433 & -0.135 & 0.620 & 0.100 & 0.440 \\
\hline 19750 & -0.134 & 0.443 & 0.107 & 0.294 & -0.132 & 0.460 & 0.140 & 0.278 \\
\hline 20000 & -0.129 & 0.307 & 0.144 & 0.186 & -0.115 & 0.240 & 0.170 & 0.171 \\
\hline 20250 & -0.110 & 0.193 & 0.191 & 0.118 & -0.0960 & 0.175 & 0.220 & 0.120 \\
\hline 20500 & -0.0925 & 0.151 & 0.271 & 0.0900 & -0.0800 & 0.130 & 0.290 & 0.0841 \\
\hline 20750 & -0.0774 & 0.118 & 0.384 & 0.0637 & -0.0680 & 0.0960 & 0.380 & 0.0658 \\
\hline 21000 & -0.0652 & 0.0954 & 0.498 & 0.0590 & -0.0570 & 0.0780 & 0.490 & 0.0558 \\
\hline 21250 & -0.0491 & 0.0618 & 0.548 & 0.0422 & -0.0420 & 0.0500 & 0.530 & 0.0415 \\
\hline 21500 & -0.0374 & 0.0414 & 0.613 & 0.0363 & -0.0280 & 0.0340 & 0.550 & 0.0274 \\
\hline 21750 & -0.0219 & 0.0253 & 0.688 & 0.0274 & -0.0200 & 0.0230 & 0.580 & 0.0202 \\
\hline 22000 & -0.0147 & 0.0153 & 0.737 & 0.0218 & -0.0139 & 0.0150 & 0.640 & 0.0177 \\
\hline 22250 & -0.00992 & 0.0104 & 0.837 & 0.0191 & -0.00800 & 0.0110 & 0.760 & 0.0150 \\
\hline 22500 & 0.00000 & 0.00000 & 1.00 & 0.0182 & 0.00000 & 0.00000 & 1.00 & 0.0136 \\
\hline 22750 & 0.00378 & -0.00189 & 0.944 & 0.0170 & 0.00500 & -0.00280 & 0.970 & 0.0130 \\
\hline 23000 & 0.00567 & -0.00149 & 0.778 & 0.0150 & 0.00700 & -0.00195 & 0.840 & 0.0122 \\
\hline 23250 & 0.00737 & -0.00113 & 0.618 & 0.0126 & 0.00940 & -0.00135 & 0.720 & 0.0106 \\
\hline 23500 & 0.00944 & -0.00078 & 0.486 & 0.0112 & 0.0106 & -0.00094 & 0.590 & 0.0102 \\
\hline 23750 & 0.0105 & -0.00056 & 0.375 & 0.00923 & 0.0113 & -0.00060 & 0.480 & 0.00995 \\
\hline 24000 & 0.00973 & -0.00041 & 0.281 & 0.00773 & 0.0109 & -0.00039 & 0.370 & 0.00822 \\
\hline 24250 & 0.00813 & -0.00030 & 0.189 & 0.00528 & 0.00990 & -0.00025 & 0.290 & 0.00684 \\
\hline 24500 & 0.00547 & -0.00020 & 0.117 & 0.00314 & 0.00810 & -0.00014 & 0.210 & 0.00519 \\
\hline
\end{tabular}

\section{Discussions}

On the assumption that the heterochromatic additivity law holds for flicker photometry, the luminous efficiency function, $V_{p}(\lambda)$ should be predicted by a linear combination of color matching functions as follows:

$$
\begin{array}{r}
V_{p}(\lambda)=l_{R} \bar{r}(\lambda)+l_{G} \bar{g}(\lambda)+l_{B} \bar{b}(\lambda) \\
\ldots \ldots \ldots \ldots \ldots \ldots
\end{array}
$$

where coefficients $l_{R}, l_{G}$, and $l_{B}$ are determined from the luminous efficiency values at the wavelengths of the respective primaries. These values were actually obtained by the flicker photometry. The coefficients are $l_{R}=0.141, l_{G}=$ 0.741 , and $l_{B}=0.0182$ for the observer $\mathrm{HY}$, and $l_{R}=0.185, l_{G}=0.757$, and $l_{B}=0.0136$ for $\mathrm{ZF}$. The predicted luminous efficiency function for each observer is shown by a solid curve in Fig. 2 . There are fairly good agreements between the measured and predicted curves (correlation coefficients were 0.9983 for the observer $\mathrm{HY}$ and 
0.9990 for $\mathrm{ZF}$ ), but the predicted curves are slightly lower than the measured ones (not exceeding 0.1 logarithmic units) in the violet wavelength region shorter than $444 \mathrm{~nm}$. This is consistent with the data obtained by Stiles-Burch ${ }^{1)}$ and Sperling ${ }^{2}$. However, taking into account the variability in both luminous efficiency and color matching measurements, these differences in the short wavelength region are not significant. Furthermore, if we are simply interested in testing whether the luminosity function by flicker photometry is a linear combination of color matching functions, then the combination of color matching functions may be determined by providing the best fit to the measured luminosity function. The dashed curves in Fig. 2 represent the synthetic functions using the coefficients determined by minimizing the squared deviations of the logarithmic values of the synthetic luminosities from those of the measured ones for each observer. The coefficients are $l_{R}=$ $0.1562, l_{G}=0.698$, and $l_{B}=0.0197$ for the observer HY, and $l_{R}=0.1825, l_{G}=0.7448$, and $l_{B}=0.015$ for $\mathrm{ZF}$. These curves provide the best possible fit to the measured curves (correlation coefficients are 0.9991 for both observers).

From the view point of making a precise color vision model however, we can not completely neglect the differences observed in the short wavelength region. There are two possible explanations for these differences. One is photometric additivity failure, that is, additivity failure for the flicker photometry; and the other is colorimetric additivity failure, that is, additivity failure of color matching functions. The former failure can be rejected because the additivity for flicker photometry has been confirmed by many independent studies. ${ }^{\text {) }}$ On the other hand, the colorimetric additivity failure has been observed by Crawford ${ }^{8)}$ and Wyszecki. ${ }^{9)}$ They carried out color matching experiments using two methods, the maximum saturation method and the Maxwell method. In the Maxwell method, one half field provided a constant white and the other half field comprised a test monochromatic stimulus and two of the three primary stimuli. Color matches were always made on the white field independent of the test wavelength. If the proportionality and additivity laws of color matching hold strictly, color matching functions using the Maxwell method should be identical to those using the maximum saturation method. However, Crawford's and Wyszecki's results showed small but systematic differences between two sets of color matching functions using both methods, particularly in the short wavelength region. The differences between the synthetic function and the measured one observed in the short wavelength region might be associated with this failure of additivity law of color matching, but further work is needed to resolve this difficult problem.

It is emphasized once again that a linear combination of color matching functions is in good agreement with the luminous efficiency function using flicker photometry. Assuming that the color matching functions are linearly related to the spectral sensitivities of the cone receptors, the present result implies that the output from the cone receptors is linearly transformed to the luminance or achromatic visual channel which is responsible for flicker photometry.

\section{Acknowledgements}

This work was carried out at National Research Council of Canada when the author stayed there as a Research Associate. He wishes to thank G. Fielder for operating the apparatus and making numerous programs for the experiment and F. Zhou for many hours of time spent as an observer. He also thanks A. R. Robertson for valuable comments on an earlier version of the manuscript.

\section{References}

(1) Stiles, W.S.: "Interim Report to the Commission Internationale de l'Eclairage, Zurich, 1955, on the National Physical Laboratory's investigation of colour matching (1955)", Opt. Acta, 2 (1955) $168-176$.

(2) Sperling, H.G.: "An experimental investigation of the relation between colour mixture and luminous efficiency." Visual problems of colour. I., National Physical Laboratory Symposium No. 8, London (1958) $251-277$.

(3) Estevez, O.: "A better colorimetric standard observer for color-vision studies: the Stiles and Burch $2^{\circ}$ color matching functions", Color Res. Appl. 7 (1982) 131- 134.

(4) Richards, W. and Luria, L.M.: "Color-mixture functions at low luminance levels", Vision Res. 4 (1964) $281-313$.

(5) Wyszecki, G. and Stiles, W.S.: Color Science, 2nd Edition, John wiley \& Sons (1982) 476 479. 
(6) Fielder, G.H.: "The NRC-Trichromator", Special Report of the Physics Division, National Research Council, Ottawa, Canada (1985).

(7) For example, Ikeda, M.: "Linearity law reexamined for flicker photometry by the summationindex method", J. Opt. Soc. Am. 73 (1983)
$1055-1061$.

(8) Crawford, B.H.: "Colour matching and adaptation", Vision Res. 5 (1965) $71-78$.

(9) Wyszecki, G.: same as Ref. 5, $379-391$.

Received December 17, 1987; accepted March 15, 1988 